\title{
Resonance Effects in Carbon Burning Process on Type la Supernovae
}

\author{
Kanji Mori ${ }^{1,2, \star}$, Michael Famiano ${ }^{3,1, \star \star}$, and Toshitaka Kajino ${ }^{1,2,4, \star \star \star}$ \\ ${ }^{1}$ National Astronomical Observatory of Japan 2-21-1 Osawa, Mitaka, Tokyo, 181-8588 Japan \\ ${ }^{2}$ Department of Astronomy, Graduate School of Science, The University of Tokyo, 7-3-1 Hongo, Bunkyo-ku, \\ Tokyo, 113-0033 Japan \\ ${ }^{3}$ Department of Physics, Western Michigan University, Kalamazoo, Michigan 49008 USA \\ ${ }^{4}$ School of Physics and Nuclear Energy Engineering, Beihang University, Beijing 100191, China
}

\begin{abstract}
The ${ }^{12} \mathrm{C}+{ }^{12} \mathrm{C}$ reaction plays an important role in type Ia supernovae. However, its cross section with astrophysical low energy has not been measured. If there is an unknown resonance in the low energy region, the reaction rate is enhanced and can affect astrophysics. We impose an upper limit to the resonance strength using the Wigner limit and find that the reaction rate can be larger than a standard rate by $\sim 10^{3}$ times in astrophysical temperature. We apply this enhanced reaction rate to binary mergers of two white dwarfs (WDs). We find that the fate of WD-WD mergers changes due to the resonance. The accretion induced collapse to neutron stars becomes more likely to occur, while it becomes more difficult to explain SNe Ia from WD-WD mergers.
\end{abstract}

\section{Introduction}

The $\mathrm{C}$ burning processes

$$
\begin{aligned}
& { }^{12} \mathrm{C}+{ }^{12} \mathrm{C} \rightarrow \alpha+{ }^{20} \mathrm{Ne}+4.6 \mathrm{MeV} \\
& { }^{12} \mathrm{C}+{ }^{12} \mathrm{C} \rightarrow \mathrm{p}+{ }^{23} \mathrm{Na}+2.2 \mathrm{MeV}
\end{aligned}
$$

plays important roles in type Ia supernovae (SNe Ia) [2], X-ray superbursts [3] and massive stellar evolution [8]. Cross sections of this reactions have been pursued by a number of experiments, but cross sections with $E<2.1 \mathrm{MeV}$ are still unknown (e.g. [4]). On the other hand, the Gamow peak at astrophysical temperature $0.5 \mathrm{GK}$ is $1.5 \mathrm{MeV}$, therefore it is necessary to explore lower energy cross sections. A theoretical study [11] on the compound nucleus ${ }^{24} \mathrm{Mg}$ suggest that there are resonances around the Gamow peak. Therefore, in this study, we assume an unknown resonance, which will enhance the reaction rate and affect astrophysics.

Binary mergers of two white dwarfs (WDs) are thought to be a progenitor of SNe Ia [5, 6], while some suggest that they end up as neutron stars (NSs) instead [14]. The fate of WD-WD mergers is dependent on the ${ }^{12} \mathrm{C}+{ }^{12} \mathrm{C}$ reaction rate (e.g. [15]) because the $\mathrm{C}$ fusion ignites the explosion. The

\footnotetext{
^e-mail: kanji.mori@nao.ac.jp

$\star \star$ e-mail: michael.famiano@wmich.edu

$\star \star \star$ e-mail: kajino@nao.ac.jp
} 

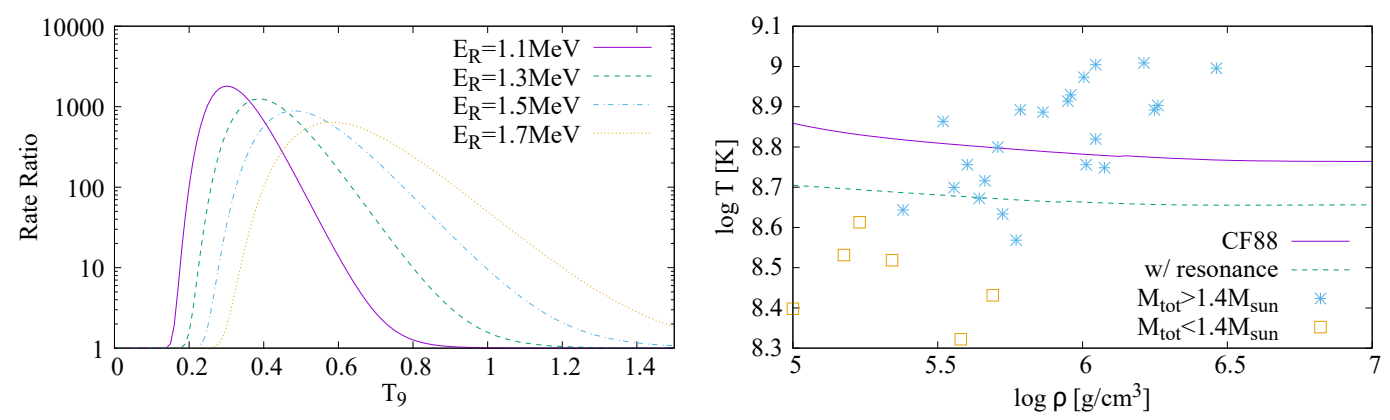

Figure 1. (Left) The upper limit of the reaction rate normalized by CF88. (Right) Ignition temperature of the ${ }^{12} \mathrm{C}+{ }^{12} \mathrm{C}$ reaction with and without the resonance. The points show the results of the SPH simulations [15].

aim of this study is to explore the effect of the low-energy resonance on the evolution of WD-WD mergers [1].

\section{Constraint on the Resonance}

The partial width of resonances must satisfy

$$
\Gamma_{\mathrm{C}}\left(E_{\mathrm{R}}\right)<2 \gamma_{\mathrm{W}}^{2} P\left(E_{\mathrm{R}}\right),
$$

where $E_{\mathrm{R}}$ is the resonance energy, $\Gamma_{\mathrm{C}}\left(E_{\mathrm{R}}\right)$ is the partial width of the incoming channel, $P(E)$ is the penetration factor and $\gamma_{\mathrm{W}}^{2}=3 \hbar^{2} /\left(2 \mu a^{2}\right)$ is the Wigner limit [7]. The penetration factor is given as

$$
P(E)=\frac{k a}{G_{l}(k a)^{2}+F_{l}(k a)^{2}},
$$

where $k$ is the wave number and $G_{l}$ and $F_{l}$ are the Coulomb wave functions. The Wigner limit depends on the channel radius $a$. Kanungo et al. [17] measured the matter radius of ${ }^{12} \mathrm{C}$ as $2.35 \mathrm{fm}$. On the other hand, the channel radius is estimated to be $a=7.97 \mathrm{fm}$ by fitting the cross section [18]. In order to estimate the upper limit of the strength, we adopt $a=7.97 \mathrm{fm}$ in this study.

Assuming spin-0, the inequality (3) can be used as the upper limit on the strength of the low energy resonances. When $E_{\mathrm{R}}=1.5 \mathrm{MeV}$, the upper limit on the resonance strength is $1.4 \times 10^{-12} \mathrm{MeV}$. Hence it is shown that some of resonances adopted in previous work $[2,3,8]$ are too strong in terms of the Wigner limit. It is also shown that known resonances listed in [9] satisfy the inequality (3). From this upper limit, the upper limit for the reaction rate is obtained. The left panel in Fig. 1 shows the upper limit for the reaction rate normalized by a standard rate [10] (hereafter CF88) as a function of $T_{9}=T /\left(10^{9} \mathrm{~K}\right)$. One can see that the resonant reaction rate can be enhanced by $\sim 10^{3}$ times compared with CF88. Chiba \& Kimura [11] suggested $E_{\mathrm{R}}=1.37 \mathrm{MeV}$ from their antisymmetrized molecular dynamics calculation on the compound nucleus ${ }^{24} \mathrm{Mg}$. We adopt this resonance energy in the next section.

\section{Implication to the Fate of WD-WD Mergers}

The fate of WD-WD binary mergers depends on relations among dynamical timescale $\tau_{\mathrm{dyn}}$, heating timescale due to $\mathrm{C}$ burning $\tau_{\mathrm{C}}=C_{P} T / \epsilon_{\mathrm{C}}$ and cooling timescale due to neutrino emission $\tau_{v}=C_{P} T / \epsilon_{v}$. 
$C_{P}$ is specific heat, $\epsilon_{\mathrm{C}}$ is the heating rate due to $\mathrm{C}$ burning and $\epsilon_{v}$ is the cooling rate by thermal neutrinos [12]. Let us assume that the total mass of the two WDs is larger than the Chandrasekhar mass $M_{\mathrm{ch}}$. If a condition $\tau_{\mathrm{C}}<\tau_{\mathrm{dyn}}$ is satisfied just after merging, the system explodes as a $\mathrm{SN}$ Ia. This scenario is called violent merger (VM; [13]). If this condition is not satisfied, the merger creates a remnant. In this phase, the system can be burnt into oxygen-neon-magnesium WD by $\mathrm{C}$ burning and collapse to a NS if $\tau_{\mathrm{C}}<\tau_{v}$. This evolutionary path is called accretion induced collapse (AIC; [14]). If this does not occur, $\mathrm{C}$ burning begins at the center and the system explodes as a SN Ia. We call this path accretion induced explosion (AIE). If the total mass is smaller than $M_{\mathrm{ch}}$, a massive WD (MWD) remains.

The right panel of Fig. 1 shows ignition temperature defined as $\tau_{\mathrm{C}}=\tau_{v}$. The points are temperature and density of the hottest points in the remnant phase in a smoothed particle hydrodynamics (SPH) simulation [15]. The resonance lowers ignition temperature, therefore more systems go to AIC, while SNe Ia become less likely to occur. WD mergers with the primary mass of $0.9 M_{\odot}$ is led to AIE with the CF88 rate, but the resonance can change their fate into AIC.

The mass distribution of WDs is estimated from Sloan Digital Sky Survey (SDSS) [16]. Galactic WD-WD merger rate is estimated as $1.4 \times 10^{-13} / M_{\odot} / \mathrm{yr}$ from SDSS [19]. One can estimate astrophysical event rates from these observations and the theoretical consideration on the fate of WD mergers. The VM rate is estimated to be $8.4 \times 10^{-17} / M_{\odot} / \mathrm{yr}$ for both of CF88 and the resonant reaction rate. The AIE rate is $1.3 \times 10^{-14} / M_{\odot} / \mathrm{yr}$ for CF88 and $8.3 \times 10^{-15} / M_{\odot} / \mathrm{yr}$ for the resonant rate. On the other hand, Galactic SN Ia rate is estimated to be $\sim 1.1 \times 10^{-13} / M_{\odot} / \mathrm{yr}$ [20]. Hence WD-WD mergers explain only $\sim 12 \%$ of SNe Ia with CF88 and $\sim 8 \%$ with the resonant rate.

The authors thank S. Kubono for helpful discussion. KM was supported by a grant from the Hayakawa Satio Fund awarded by the Astronomical Society of Japan.

\section{References}

[1] Mori, K. Master Thesis (The University of Tokyo); to be submitted to ApJ

[2] Bravo, E. et al. A\&A 535, A114 (2011)

[3] Cooper, R. L., Steiner, A. W., \& Brown, E. F. ApJ 702, 660 (2009)

[4] Spillane, T. et al. PRL 98, 122501 (2007)

[5] Iben, I. Jr., \& Tutukov, A. V. ApJS 54, 335 (1984)

[6] Webbink, R. F. ApJ 277, 355 (1984)

[7] Teichmann, T., \& Wigner, E. P. PR 87, 123 (1952)

[8] Bennett, M. E. et al. MNRAS 420, 3047 (2012)

[9] Aguilera, E. F. et al. PRC 73, 064601 (2006)

[10] Caughlan, G. R., \& Fowler, W. A. ADNDT 40, 283 (1988)

[11] Chiba, Y., \& Kimura, M. PRC 91, 061302 (2015)

[12] Itoh, N. et al. ApJS 102, 411 (1996)

[13] Pakmor, R. et al. Nature 463, 61 (2010)

[14] Nomoto, K., \& Kondo, Y. ApJ, 367, L19 (1991)

[15] Sato, Y. et al. ApJ 807, 105 (2015)

[16] Kepler, S. O. et al. MNRAS 375, 1315 (2007)

[17] Kanungo, R. et al. PRL 117, 102501 (2016)

[18] Yakovlev, D. G. et al. PRC 82, 044609 (2010)

[19] Badenes, C., \& Maoz, D. ApJ 749, L11 (2012)

[20] Li, W. et al. MNRAS 412, 1473 (2011) 